\title{
MaTGeC: hacia un marco de trabajo para la gestión cuantitativa de procesos de desarrollo de software en pequeñas organizaciones
}

\author{
MaTGeC: Towards A Framework For Quantitative Process \\ Management Of Software Development In Very Small Entities
}

\author{
Carlos Alberto Ardila Albarracín \\ Ingeniero de sistemas, magíster en Computación, docente de la Universidad del \\ Cauca, Popayán, Colombia \\ Contacto: cardila@unicauca.edu.co
}

Francisco José Pino Correa

Ingeniero en Electrónica y Telecomunicaciones, doctor en Ingeniería de Sistemas, docente de la Universidad del Cauca, Popayán, Colombia.

Contacto:fjpino@unicauca.edu.co

\section{César Jesús Pardo Calvache}

Ingeniero de Sistemas, Doctor y Magister en Ingeniería Informática, Docente de la Universidad EAFIT, Medellín, Colombia

Contacto:cpardoc@eafit.edu.co

\section{Luis Merchán PARedes}

Ingeniero de sistemas, magíster en Administración de Empresas, doctor en Dirección de Proyectos, director de Investigaciones de la Universidad de San Buenaventura, Cali, Colombia.

Contact: :lmerchan@usbcali.edu.co

Fecha de recepción: : 31 de mayo de 2013

Fecha de aceptación: 16 de mayo de 2014

Clasificación del artículo: revisión de tema Financiamiento: Universidad de San Buenaventura de Cali

Palabras clave: gestión cuantitativa de procesos, ingeniería de software, desarrollo de software, organizaciones pequeñas

Keywords: quantitative process management, software engineering, software development, small entities 


\section{revisión}

\section{RESUMEN}

Las organizaciones pequeñas desarrolladoras de software tienen dificultades para adoptar las prácticas sobre gestión cuantitativa de procesos ofrecidas por modelos de referencia como CMMIDEV o ISO/IEC 15504, debido a que les generan costosas inversiones a largo plazo. Sin embargo, en caso de que no incorporen al menos las prácticas esenciales, no podrán obtener procesos con menor variabilidad en su rendimiento y que puedan estar bajo control estadístico, lo que afecta de manera directa en el resultado de sus proyectos, ya que permitiría efectuar mejores estimaciones. En este artículo se presenta una propuesta general de un marco de trabajo para gestión cuantitativa de procesos para pequeñas organizaciones (MaTGeC) que ofrece una guía para monitorear y evaluar procesos específicos seleccionados por la organización. Posteriormente, se indican los componentes que lo conforman, junto con las prácticas, actividades, roles y productos esenciales para que esta clase de organizaciones incorporen los fundamentos de gestión cuantitativa en sus tareas más críticas.

\section{ABSTRACT}

The small software development companies have difficulties in adopting practices about quantitative process management offered by reference models such as CMMI-DEV or ISO/IEC 15504 because it generates expensive investments in long-term periods of time. However, if they do not incorporate at least the essential practices, they won't be able to obtain processes with less variability in its performance and that can be statistically under control which directly impacts the projects results because they would allow making better estimates. In this article, it is presented a general proposal of a framework for quantitative process management in very small entities (MaT$\mathrm{GeC}$ ) that offers a guide to monitor and assess specific processes selected by the organization. Moreover, it is indicated the components that conform it, along with the practices, activities, roles and essential work products for that this kind of organizations to incorporate the quantitative management fundamentals in their more critical tasks.

\section{INTRODUCCIÓN}

Las organizaciones desarrolladoras de software abordan planes de mejora de procesos para alcanzar un mayor grado de calidad en sus procesos y productos. La mejora de procesos de desarrollo de software implica seguimiento continuo de procesos para predecir su comportamiento y sus variaciones de rendimiento (Baldassarre, 2004). En un plan de mejora se requiere evaluar diversos atributos de un proceso, porque de no recolectar ni analizar información al respecto, será imposible controlar un proceso desde un punto de vista estadístico. En consecuencia, las organizaciones requieren gestionar de manera cuantitativa sus procesos de desarrollo de software por medio de

la aplicación de técnicas estadísticas (Díaz-Ley, 2008). La gestión cuantitativa de procesos (GCP) proporciona una visión del grado de cumplimiento de metas así como las causas profundas que explican desviaciones significativas tanto en los procesos como en los productos (Gou, 2009).

Por otra parte, debido a que las pequeñas organizaciones son mayoría en la industria del software - p. ej., en Colombia conforman un $89 \%$ (Fedesoft, 2010)_, se considera importante conocer y analizar los enfoques existentes sobre gestión cuantitativa de procesos software enfocados en este tipo de organizaciones. Conocer, disponer y utilizar estos enfoques fortalecería la industria del software nacional. 


\section{revisión}

Ahora bien, es cierto que existen varios modelos de referencia para la mejora y evaluación de los procesos de desarrollo software, como el modelo de madurez de la capacidad integrado (CMMI en sus siglas en inglés) y más específicamente CMMI para Desarrollo - CMMI-DEV en inglés - (CMMI Product Team, 2010), ISO/ IEC 15504-5 (ISO/IEC 15504-5, 2006) e ISO/ IEC 12207 (ISO/IEC 12207, 2008). Sin embargo, debido a que esos modelos han sido orientados a las grandes organizaciones, junto al hecho de que muy pocos estudios han centrado su interés en el uso de las prácticas efectivas hacia las características de las pequeñas organizaciones, el conocimiento de los modelos por parte de estas ha sido débil (Calvo-Manzano, 2008). Además, de acuerdo con Mas y Amengual (2005), para una pequeña organización es muy costoso desarrollar y reflejar los resultados de la implantación de programas de mejora con el nivel de detalle y formalidad que acostumbran a exigir los modelos orientados a grandes empresas. Otro problema radica en que el retorno de la inversión de un programa de mejora de procesos no se produce en el corto plazo. Por lo anterior, Mas y Amengual (2005) afirman que la dificultad de aplicación de los grandes modelos de mejora se debe a los costes asociados a su aplicación y al largo plazo de espera necesario para la observación de resultados.

Además, aunque se han elaborado modelos de referencia para pequeñas organizaciones, como MoProSoft (NYCE, 2011), Competisoft (Competisoft, 2008) y MPS.BR (Melhoria de Processo do Software Brasileiro, 2005-2012), estos no ofrecen elementos suficientes para efectuar gestión cuantitativa de procesos de desarrollo de software en esta clase de organizaciones.

Los aspectos antes mencionados han dificultado la adopción de una cultura de gestión cuantitativa en el interior de estas organizaciones, lo que ha derivado en un problema: una pequeña organización desarrolladora de software que no adopte prácticas de gestión cuantitativa, tendrá dificul- tades para identificar y enfocarse en los factores que impactan en el rendimiento de los procesos y por consiguiente en los productos que pudieran obtenerse a partir de ellos.

En este artículo se pretende enfrentar ese problema proponiendo un marco de trabajo para gestión cuantitativa de procesos para pequeñas organizaciones (MaTGeC), el cual incluye elementos que se ubican en un estado posterior al de efectuar mediciones, es decir, el enfoque es de análisis de datos, en lugar de recolectarlos, con el fin de obtener procesos de desarrollo de software que se caractericen por tener una variabilidad menor y que estén bajo control, desde un punto de vista estadístico.

El artículo tiene la siguiente estructura: en la segunda sección se explica el método utilizado para la elaboración de la propuesta, en la tercera sección se muestran los resultados, que están representados en las características y los componentes de MaTGeC. Finalmente, en la cuarta sección se presentan las conclusiones.

\section{METODOLOGÍA}

En esta sección se presenta la estructura del método utilizado para elaborar la propuesta de MaTGeC. También se indican los trabajos relacionados a partir de los cuales se hace posible identificar la necesidad que da origen a MaTGeC.

\section{Método utilizado}

MaTGeC fue el resultado de la ejecución de algunas de las tareas propuestas por el Método de Producción del OPEN Process Framework (Opfro, 2009), que es de dominio público y se utiliza para la producción de métodos de desarrollo específcos. Es importante hacer uso de un método como este, porque se considera necesario que $\mathrm{MaTGeC}$ sea el resultado de seguir directrices confiables, planteadas por organizaciones reconocidas en el 


\section{revisión}

área de mejora de procesos. El objetivo es que la propuesta que se presente tenga bases teóricas sólidas, al mismo tiempo que refleje buenas prácticas en la elaboración de métodos, las cuales ya han sido probadas y aceptadas de manera amplia en la industria de software.

Ahora bien, la propuesta de MaTGeC es el producto principal de una tesis de maestría, de modo que esta circunstancia tiene como consecuencia que el equipo de trabajo sea muy reducido. Por lo anterior, ha sido necesario seleccionar cinco de las nueve tareas sugeridas por el método de producción. Las tareas elegidas para la elaboración de los componentes de MaTGeC son las siguientes:

a. Evaluación de necesidades sobre MaTGeC. Se evalúan las necesidades específicas relacionadas con gestión cuantitativa de procesos, las cuales se espera abordar con los elementos que se van a incluir en la propuesta.

b. Construcción de MaTGeC. El equipo de trabajo construye el marco de trabajo por medio de la selección de elementos de proceso incluidos en repositorios existentes, de la adaptación de esos elementos seleccionados y de la integración de los elementos adaptados a la propuesta.

c. Documentación de MaTGeC. El equipo de trabajo registra los documentos de descripción de los elementos constitutivos del marco de trabajo.

d. Consultoría (evaluación). El equipo de trabajo acude a personal de reconocida trayectoria para que evalúe la propuesta del marco de trabajo, presente las observaciones y sugiera las correcciones que considere necesarias.

e. Mantenimiento. El equipo de trabajo procede a agregar, eliminar o modificar componentes del marco de trabajo como resultado de las revisiones derivadas de la actividad de consultoría.

\section{Trabajos relacionados}

En esta sección se analizan los aportes de diversos artículos sobre gestión cuantitativa de procesos de desarrollo de software. A partir de lo encontrado en esos artículos se identifica la necesidad que da origen a MaTGeC, de la cual se indican sus aspectos más importantes.

Tarhan (2006) señala que existen enfoques para la evaluación de los procesos software y las métricas que es preciso tener en cuenta para iniciar la implementación de gráficos de control, los cuales son una técnica importante del control estadístico de procesos (CEP). Los requerimientos básicos para implementar el uso de esos gráficos son:

a. Muestreo racional de ejecución de procesos y datos, junto con,

b. Utilización de datos de métricas para análisis estadístico.

Tarhan (2006) expresa que al establecer directrices para muestreo racional y utilización de métricas, una organización puede aplicar técnicas de CEP y así alcanzar la capacidad de entender sus procesos basados en datos cuantitativos.

Asimismo, Vijaya (2010) presenta los resultados obtenidos a través de un estudio de caso, en donde analiza datos de siete proyectos, y concluye que los gráficos de control son eficientes en el mantenimiento de la calidad de software y pueden utilizarse por parte de organizaciones desarrolladoras de software con niveles de madurez 1 o 2 de CMMI-DEV.

Adicionalmente, Gou (2007) reporta un método empírico para identificar objetivos de rendimiento, definir una línea base de rendimiento y establecer un modelo de gestión cuantitativa para procesos de prueba de software. Esa propuesta se extiende en el artículo de Gou (2008) donde presenta el método BiDefect (process-performance 


\section{revisión}

Baselines based iteration Defect management), el cual se elaboró para dar soporte a la gestión cuantitativa de defectos en desarrollo iterativo. Así mismo, Gou (2008) reporta una experiencia industrial con la empresa china de telecomunicaciones ZZNode Technologies Company Limited, que aplicó exitosamente el método BiDefect para estimación, análisis, re-estimación, y control de defectos para proyectos de desarrollo de carácter iterativo.

También están disponibles iniciativas sobre gestión cuantitativa de procesos de desarrollo de software, en las que adaptan prácticas de Six Sigma, como se indica a continuación:

- Modelo de procesos de ingeniería de software. En el trabajo de Xiaosong (2008) se hace uso de la herramienta DMAIC (Define, Measure, Analyze, Improve, Control), la cual hace parte de la metodología Six Sigma, para establecer un modelo de procesos de ingeniería de software. Ese modelo está conformado por: a) el modelo de gestión de procesos de software basado en DMAIC; b) el modelo de procesos de ingeniería de requerimientos basado en IDOV (Identify, Design, Optimize, Verify); c) herramientas y métodos de Six Sigma para gestión de calidad. Los resultados obtenidos indican que el método es factible para soportar gestión de calidad de software (Xiaosong, 2008).

- Método de gestión y análisis de proyectos. Resultado de una investigación sobre la conjunción de métodos de gestión Six Sigma y tecnologías relacionadas (Lin, 2009).

- Validación de instrumentos para la implementación de Six Sigma. El artículo de Deshmukh (2009) presenta un estudio orientado al desarrollo y validación de un instrumento para la implementación de Six Sigma en pequeñas y medianas organizaciones de la India. Se identifican 12 factores críticos en Six Sigma y se indican los 42 ítems del instrumento de implementación. Se plantea que las organizaciones pueden usar estas medidas de manera individual o generar un esquema de prácticas Six Sigma para su uso en toda la organización (Deshmukh, 2009).

- Personal Software Process (PSP) y Six Sigma. La utilización de PSP junto con Six Sigma permite analizar datos y mejorar sistemáticamente el rendimiento del proceso. Se relacionan las herramientas Six Sigma con cada proceso de PSP para mostrar que las técnicas Six Sigma pueden aplicarse a datos de PSP e indicar el uso práctico de las guías Six Sigma para soportar actividades de mejora de procesos tanto individual como grupalmente (Park, 2006). Ese trabajo tiene como continuación el desarrollo de un framework que guía cómo y dónde se enfocan las herramientas Six Sigma dentro de PSP o Team Software Process (TSP). Se concluye que el aumento de rendimiento puede alcanzarse al identificar problemas y analizar medidas, lo que provee fundamento para aplicar Six Sigma en un proyecto completo o en toda la organización (Park, 2007).

\section{Síntesis}

A partir de lo anterior, se destacan trabajos en los que se plantean y aplican métodos propios para efectuar gestión cuantitativa y lo referente a trabajos donde se recopilan estudios sobre adaptación de la metodología Six Sigma en programas de mejora de procesos de desarrollo de software. Los artículos que implementan propuestas para gestión cuantitativa de procesos de desarrollo de software, abordan aspectos particulares como la gestión de defectos, la gestión de riesgos o pruebas de software. También se encuentran estudios en los que abordan elementos conceptuales y técnicos de GCP y los enlazan con las actividades de nivel estratégico o con las metas de negocio de las organizaciones. 


\section{revisión}

Por otra parte, se observa que algunas de las propuestas están alineadas con modelos de referencia como CMMI-DEV; sin embargo, se encuentra que no está disponible una propuesta que incorpore prácticas y técnicas que apoyen la gestión cuantitativa de procesos de desarrollo de software enfocada a pequeñas organizaciones.

En este sentido, se pretende enfrentar esa situación al proponer un marco de trabajo que facilite la incorporación de prácticas de GCP en pequeñas organizaciones desarrolladoras de software, de modo que puedan reflejar resultados de la implantación de esas prácticas en el corto plazo y con una inversión en recursos que sea ostensiblemente menor que la requerida por modelos de referencia para la mejora y evaluación de procesos de desarrollo de software como son CMMI-DEV (CMMI Product Team, 2010), ISO/IEC 15504-5 (ISO/IEC 15504-5, 2006) e ISO/IEC 12207 (ISO/ IEC 12207, 2008).

\section{RESULTADOS}

En esta sección se presenta el resultado materializado en la propuesta de MaTGeC, inicialmente desde un punto de vista general y posteriormente especificando sus características.

\section{Generalidades de MaTGeC}

MaTGeC incluye los elementos que se relacionan a continuación:

a. Un componente de guía técnica para GCP. De manera general, describe los roles involucrados, las prácticas y las actividades que es necesario efectuar y los productos que se van a generar. De manera particular, ofrece una guía concreta de evaluación y monitoreo de cada proceso seleccionado por la organización para valorar la capacidad de ese proceso e identificar los factores que inciden en su variabilidad, además de saber si cumple las metas planteadas para esas características de variabilidad y capacidad, con el fin de conceptuar si ese proceso se halla estadísticamente bajo control.

b. Un componente de técnicas para control estadístico de procesos y para análisis estadístico. Incluye fichas descriptivas sobre las técnicas estadísticas necesarias para ejecutar las prácticas y actividades del primer componente.

c. Un componente de documentos y plantillas para orientar el uso de las técnicas del segundo componente y registrar lo requerido por el primero de ellos.

La figura 1 presenta la vista general de MaTGeC.

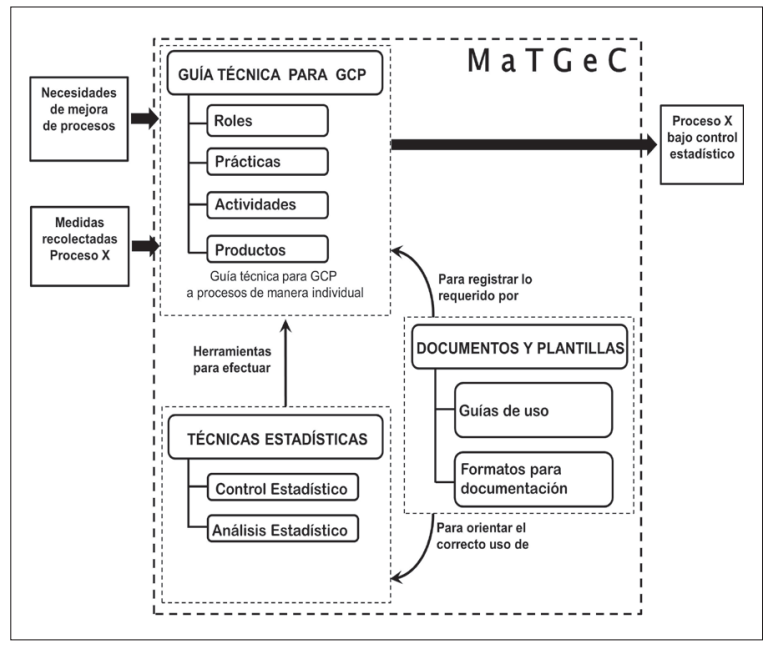

Figura 1. Vista general de MaTGeC

Fuente: elaboración propia.

MaTGeC presenta las siguientes características:

a. Orientado a pequeñas organizaciones. La propuesta incluye pocas actividades y productos, buscando que el personal de la organización no tenga que incrementar de manera significativa el esfuerzo necesario al momento de utilizar las técnicas asociadas y generar los productos correspondientes. Sin embargo, una pequeña empresa que desee implementar 


\section{revisión}

las prácticas de MaTGeC debe tener un nivel alto de madurez que le permita ejecutar de manera correcta los siguientes procesos:

i. Gestión de requisitos. La organización maneja un único formato para documentar cada requisito, de tal forma que sean sencillos de entender y que muestren trazabilidad con las fuentes de obtención, con otros requisitos y con otros productos de trabajo. Además se asegura que se revise la información contenida en los ejemplares de ese formato para detectar inconsistencias.

ii. Planificación de proyecto. La empresa tiene adoptados métodos para estimaciones en cuanto a costos, cronogramas y personal requerido para un proyecto; tiene asimismo definido el modelo de ciclo de vida y efectúa gestión de riesgos. Todos esos ítems los documenta en el plan de proyecto.

iii. Monitoreo y control de proyecto. Se hacen revisiones de cada área del proyecto, con periodos de tiempo cortos (menores a 20 días), y se registra el resultado de esas revisiones en un documento que informa sobre las inconsistencias encontradas y el grado de avance.

iv. Gestión de la configuración. La organización realiza control de versiones de los códigos fuentes y de cada uno de los documentos y almacena un historial de cambios realizados sobre ellos, identificando el responsable de dichos cambios.

b. Directrices técnicas para obtener un proceso gestionado cuantitativamente. Esta característica hace posible que los usuarios de MaT$\mathrm{GeC}$ puedan seguirlo correctamente para llevar al proceso en cuestión al estado deseado. Esto se logra a partir de la información que MaTGeC incluye sobre cómo usar las herramientas de control estadístico de procesos, las técnicas de análisis estadístico y los productos, para determinar con un esfuerzo razonable, los objetivos de calidad y rendimiento del proceso en la organización así como las líneas base de rendimiento del proceso.

\section{Descripción de MaTGeC}

Propósito. Guiar la implementación de prácticas y técnicas para efectuar GCP en el contexto de las pequeñas organizaciones, con el fin de obtener procesos con menor variabilidad y estadísticamente bajo control. MaTGeC tiene como objetivos:

a. Establecer los elementos necesarios para guiar las prácticas de GCP en una pequeña organización de desarrollo de software, buscando la obtención de procesos controlables y estadísticamente predecibles en el interior de la organización.

b. Facilitar su aplicación en pequeñas organizaciones de desarrollo de software, al definir una cantidad reducida de prácticas, actividades y productos.

Componente de guía técnica para GCP. MaT$\mathrm{GeC}$ ofrece una guía para gestionar, monitorear y evaluar procesos específicos seleccionados por la organización. Cada proceso que se desee gestionar cuantitativamente debe tener como entrada las medidas de la característica de desempeño del proceso (p. ej., si el proceso es codificación a escala unitaria, es útil tener datos sobre densidad de defectos). MaTGeC indica que para cada proceso debe ejecutarse un conjunto de actividades agrupadas en cuatro grandes prácticas:

a. Valoración inicial de desempeño de un proceso

b. Análisis causal

c. Preparación para gestión cuantitativa

d. Ejecución de gestión cuantitativa 


\section{revisión}

Las prácticas han sido especificadas a partir de la revisión de los elementos esenciales de las prácticas de gestión cuantitativa indicadas en los modelos para mejora y evaluación de procesos de desarrollo software de mayor uso como CMMI-DEV (CMMI Product Team, 2010) e ISO/IEC 15504 (ISO/IEC 15504:2006). Los modelos mencionados se tuvieron en cuenta solo como referencia respecto al panorama general de las prácticas asociadas con gestión cuantitativa y la interacción entre ellas. Las prácticas incluidas en MaTGeC se consideran las mínimas que es necesario ejecutar y se han elegido teniendo en cuenta el perfil de las pequeñas organizaciones y el tipo de proyectos que manejan, por lo que requieren prácticas que impliquen calidad y eficiencia cuando se requiera gestionar de manera cuantitativa los procesos que considere más importantes y para que alcancen metas establecidas para esos procesos en el corto plazo.
En la figura 2 se muestra el diagrama general de actividades de MaTGeC, las cuales se deben aplicar para cada proceso seleccionado por la organización.

Pensando en que deben ser pocos los roles involucrados a causa del bajo número de personal disponible en las pequeñas empresas, MaTGeC plantea involucrar los roles ya existentes (analista, diseñador, programador, tester, gestor de calidad), los cuales se denominan de manera genérica "responsable de proceso", de modo que participen de manera activa pero sin ocasionar una excesiva carga adicional a sus responsabilidades. Por esto, se plantea como único rol nuevo que incluir el "agente de gestión cuantitativa", cuyas responsabilidades se presentan en la tabla 1. Este rol debe tener las siguientes características mínimas: profesional en ingeniería de sistemas enfocado a

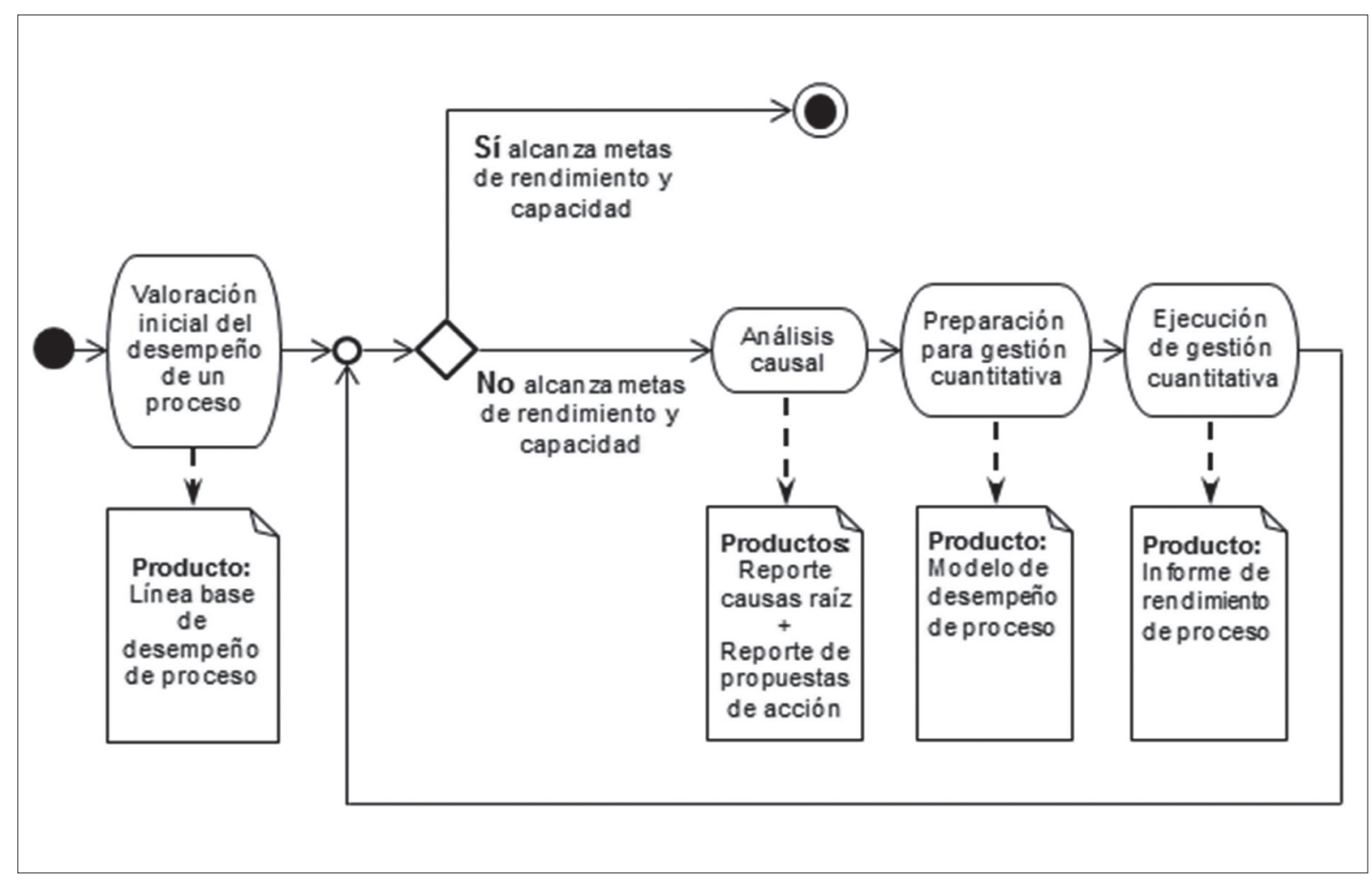

Figura 2. Diagrama general de prácticas y productos de MaGTeC para procesos seleccionados

Fuente: elaboración propia. 
la ingeniería de software, con conocimientos en modelos de mejora de procesos de software, estadística básica, técnicas de control estadístico de procesos y técnicas de análisis estadístico. Además, el "responsable de proceso" debe suministrar la información necesaria para que el "agente de gestión cuantitativa" pueda generar los productos indicados.

Componente de técnicas estadísticas. Este componente contiene guías de referencia que presentan información resumida sobre las técnicas estadísticas requeridas para ejecutar las prácticas y actividades de MaTGeC. En cuanto a técnicas de CEP se han incluido: i) diagramas de control y ii) diagramas causa-efecto o "espina de pescado", y en cuanto a técnicas de análisis estadístico se eligieron: i) análisis de regresión y ii) análisis de capacidad de proceso. Su inclusión se justifica por el hecho de que el CEP hace uso de gráficos de control, junto con otras técnicas estadísticas como el análisis de Pareto (Radice, 2000). Además de las ya mencionadas, también se sugiere el uso del análisis de regresión y diagramas causaefecto (Xiaosong, 2008; Lin, 2009).

Por otra parte, la justificación para incluir la técnica denominada "análisis de capacidad de proceso" es la siguiente: "deben incluirse índices de capacidad (de proceso) asociados con la variación a corto plazo junto con los índices asociados con la variación a largo plazo; por ejemplo, con CMMI-DEV se suele considerar que 1.33 es el valor mínimo aceptable para un índice de capacidad, debido a que cualquier valor por debajo de esta cifra indicaría que aunque el proceso esté bajo control estadístico, no cumple con las especificaciones deseadas" (Pérez, 2012).

Componente de documentos y plantillas. Se divide en dos partes: la primera contiene una guía de uso con un ejemplo completo en el que se muestra paso a paso cómo ejecutar cada una de las actividades que integran las prácticas de la presente propuesta, además de cómo documentarlas. La segunda parte incluye los formatos nece-

Tabla 1. Responsabilidades del rol "agente de gestión cuantitativa"

\begin{tabular}{|c|c|}
\hline Práctica & $\begin{array}{l}\text { Responsabilidad } \\
\text { (representa las actividades asociadas a las prácticas) }\end{array}$ \\
\hline $\begin{array}{l}\text { Valoración inicial de desempeño } \\
\text { de un proceso }\end{array}$ & $\begin{array}{l}\text { Definir medidas de desempeño de proceso con la justificación de su selección, incluyendo } \\
\text { trazabilidad a otros procesos } \\
\text { - } \quad \text { Analizar y reportar datos de desempeño de proceso } \\
\text { - } \quad \text { Elaborar el modelo de desempeño de proceso }\end{array}$ \\
\hline Análisis causal & $\begin{array}{l}\text { - } \quad \text { Generar reporte de resultados del análisis de causas raíz } \\
\text { - } \quad \text { Establecer propuestas de acción para solucionar causas } \\
\text { - } \quad \text { Elaborar informes sobre análisis de desempeño de proceso y del cambio en tal desempeño } \\
\text { - } \quad \text { Consolidar registros de análisis causal }\end{array}$ \\
\hline $\begin{array}{c}\text { Preparación para gestión } \\
\text { cuantitativa }\end{array}$ & $\begin{array}{l}\text { - } \quad \text { Definir objetivos de calidad y de desempeño de proceso } \\
\text { - } \quad \text { Elaborar reportes de subprocesos y atributos seleccionados } \\
\text { - Establecer la línea base de desempeño de proceso }\end{array}$ \\
\hline Ejecución de gestión cuantitativa & $\begin{array}{l}\text { - } \quad \text { Elaborar reportes sobre límites de desempeño de proceso } \\
\text { - Generar informes de calidad o rendimiento de proceso respaldados en técnicas de control } \\
\text { estadístico y de análisis estadístico }\end{array}$ \\
\hline
\end{tabular}

Fuente: elaboración propia. 


\section{revisión}

sarios para registrar la información requerida en el componente de guía técnica.

Soporte tecnológico. En la tabla 2 se indican productos software que apoyan la aplicación de las técnicas estadísticas mencionadas.

\section{Guía de uso}

Se ha hecho una aplicación a modo de ejemplo en el que se muestra paso a paso cómo realizar las prácticas definidas en la guía técnica, aplicadas al proceso de "construcción de software", de acuerdo con la definición de la norma ISO 12207 (ISO12207:2008, p. 63). Es de resaltar que un proceso puede evaluarse a partir de varios atributos; sin embargo, para facilitar el seguimiento de este ejemplo solo se evalúa el atributo "cantidad de defectos encontrados". También se indica en esa guía cómo deben elaborarse los productos haciendo uso de los formatos incluidos en el componente de documentos y plantillas.

\section{CONCLUSIONES}

En este artículo se ha identificado la necesidad que tienen las pequeñas organizaciones desarrolladoras de software de llevar a cabo la definición e implementación de prácticas de gestión cuantitativa en sus procesos. Adoptar esas prácticas haría factible identificar factores que impactan en el rendimiento de sus procesos y por consiguiente en los productos que pudieran obtenerse a partir de ellos. Teniendo en cuenta lo anterior, se ha

Tabla 2. Productos software de herramientas estadísticas

\begin{tabular}{|c|c|c|}
\hline Producto & Características y técnicas incluidas & $\begin{array}{c}\text { Práctica de MatGeC } \\
\text { que puede apoyar }\end{array}$ \\
\hline $\begin{array}{c}\text { Statistical Package for } \\
\text { Social Science (SPSS) } \\
\text { Versión } 20\end{array}$ & $\begin{array}{l}\text { Control estadístico de calidad, series temporales, análisis de regresión (IBM } \\
\text { SPSS Statistics 20, 2011) }\end{array}$ & Práctica 3 \\
\hline Minitab Versión 16 & $\begin{array}{l}\text { Análisis de regresión, diseño de experimentos, CEP, análisis de sistemas de } \\
\text { medición, análisis de confiabilidad, series de tiempo, simulación y distribuciones } \\
\text { (Minitab Inc., 2012) }\end{array}$ & $\begin{array}{l}\text { Práctica } 1 \\
\text { Práctica } 2 \\
\text { Práctica } 3 \\
\text { Práctica } 4\end{array}$ \\
\hline GenStat Versión 14 & $\begin{array}{l}\text { Análisis de regresión lineal, análisis de series de tiempo, métodos para CEP } \\
\qquad(\text { VSN International, 2012) }\end{array}$ & $\begin{array}{l}\text { Práctica } 1 \\
\text { Práctica } 2 \\
\text { Práctica } 3 \\
\text { Práctica } 4\end{array}$ \\
\hline OpenStat & $\begin{array}{l}\text { Herramienta gratuita. Incluye análisis bivariado (medidas de correlación y } \\
\text { regresión), análisis multivariante (correlación múltiple), elementos para CEP y } \\
\text { elementos básicos para simulación (Miller, 2013) }\end{array}$ & $\begin{array}{l}\text { Práctica } 1 \\
\text { Práctica } 2 \\
\text { Práctica } 3 \\
\text { Práctica } 4\end{array}$ \\
\hline
\end{tabular}

Fuente: elaboración propia. 


\section{revisión}

propuesto un marco de trabajo que facilita la incorporación de prácticas de GCP para esta clase de organizaciones.

Como parte de la definición de esta propuesta, se han detallado los componentes de MaTGeC: i) un componente que guía técnicamente la gestión cuantitativa de procesos de desarrollo de software, ii) un componente de técnicas para control estadístico de procesos y de métodos para análisis estadístico, iii) un componente de documentos y plantillas. También se describieron algunos elementos de proceso como propósito, objetivos, actividades, roles, productos y el soporte tecnológico que integran la propuesta.

Actualmente se está trabajando en la definición de las actividades y productos que hacen parte del componente de guía técnica. Además de lo anterior, se está elaborando la guía de uso que incluye información para orientar la aplicación de las técnicas para CEP y de las técnicas de análisis estadístico que hacen parte del módulo de técnicas. Igualmente, se están elaborando los formatos que es necesario incluir en el componente de documentos y plantillas.

\section{FINANCIAMIENTO $Y$ AGRADECIMIENTOS}

Este artículo es el resultado del trabajo de una pasantía de investigación realizada en la Universidad de San Buenaventura de Cali, como parte de las actividades curriculares de la Maestría en Computación de la Universidad del Cauca. Carlos A. Ardila agradece a la Universidad de San Buenaventura por el apoyo brindado durante esa pasantía e igualmente agradece a la Universidad del Cauca, donde trabaja como profesor asociado, por el apoyo brindado para realizar sus estudios de Maestría en Computación. Francisco J. Pino agradece a la Universidad del Cauca, donde trabaja como profesor titular. César Jesús Pardo Calvache agradece a la Universidad EAFIT donde se desempeña como Docente. Luis Merchán Paredes agradece a la Universidad de San Buenaventura donde trabaja como docente.

\section{REFERENCIAS}

Baldassarre, T., Boffoli, N., Caivano, D. y Visaggio, G., "Managing Software Process Improvement (SPI) through Statistical Process Control (SPC)", Lecture Notes in Computer Science, Vol. 3009, 2004, pp. 30-46.

Calvo-Manzano, J., García, I. y Arcilla, M., "Hacia la gestión cuantitativa en la gestión de proyectos en el ámbito de las PyMES", REICIS Revista Española de Innovación, Calidad e Ingeniería del Software, Vol. 4, No. 2, 2008, pp. 7-19.
CMMI Product Team, CMMI for Development version 1.3 Technical Report, Software Engineering Institute, 2010.

Competisoft, Mejora de procesos para fomentar la competitividad de la pequeña y mediana industria del software de Iberoamérica, 2008. Recuperado de http://goo.gl/4ji05

Deshmukh, S. y Lakhe, R., "Development and Validation of an Instrument for Six Sigma Implementation in Small and Medium Sized Enterprises", $2^{\text {nd }}$ International 
Conference on Emerging Trends in Engineering and Technology, ICETET, 2009.

[Díaz-Ley, M., García, F. y Piattini, M., "Implementing a Software Measurement Program in Small and Medium Enterprises: A Suitable framework", IET Software, Vol. 2, No. 5, 2008, pp. 417-36.

Fedesoft, Sector de TI en Colombia año 2010, Federación Colombiana de la Industria del Software y Tecnologías Informáticas Relacionadas, 2010.

Gou, L., Wang, Q., Jiang, N., Che, M., Zhang, R., Yang, Y. y Li, M., “An Empirical Study on Establishing Quantitative Management Model for Testing Process", Lecture Notes in Computer Science, Vol. 4470, 2007, pp. 233-45.

Gou, L., Wang, Q., Yuan, J., Yang, Y., Li, M. y Jiang, N., "Quantitatively Managing Defects for Iterative Projects: An Industrial Experience Report in China", Lecture Notes in Computer Science, Vol. 5007, 2008, pp. 369-80.

Gou, L., Wang, Q., Yuan, J., Yang, Y., Li, M. y Jiang, N., "Quantitative Defects Management in Iterative Development with BiDefect", Software Process Improvement and Practice, Vol. 14, No. 4, 2009, pp. 227-41.

IBM Corporation, Manual del usuario del sistema básico de IBM SPSS Statistics 20, 2011. Recuperado de http://goo.gl/CSXgx

ISO/IEC 12207, Systems and Software Engineering-Software Life Cycle Processes, Ginebra: International Organization for Standardization, 2008.
ISO/IEC 15504-5, Information TechnologyProcess Assessment-Part 5: An Exemplar Process Assessment Model, Ginebra: International Organization for Standardization, 2006.

Lin, M., Cai, C. y Li, J., "Realization and Research of a Six-Sigma-Based Project Management and Analysis System", IFCSTA 2009 Proceedings - 2009 International Forum on Computer Science-Technology and Applications, Vol. 3, 2009, pp. 70-73.

Mas, A. y Amengual, E., "La mejora de los procesos de software en las pequeñas y medianas empresas (PYME). Un nuevo modelo y su aplicación a un caso real", REICIS, Revista Española de Innovación, Calidad e Ingeniería del Software, Vol. 1, No. 2, 2005, pp. 7-29.

Melhoria de Processo do Software Brasileiro, Guia Geral MPS de Software, 2005-2012.

Miller, B., Free Statistics Programs and Materials by Bill Miller, 2013. Recuperado de http://goo.gl/L3qyk

Minitab, Características de Minitab Statistical Software, 2012. Recuperado de http://goo. $\mathrm{gl} / \mathrm{S} 2 \mathrm{eHZ}$

NYCE， Norma NMX-I-059/02-NYCE-2011 (Moprosoft), 2011. Recuperado de http:// goo.gl/9phyp

OPFRO, OPEN Process Framework Repository Organization, 2009. Recuperado de http:// goo.gl/iC3tu

Park, Y., Choi, H. \& Baik, J., “A Framework for the Use of Six Sigma tools in PSP/TSP”, 


\section{revisión}

Proceedings - SERA 2007: Fifth ACIS International Conference on Software Engineering Research, Management, and Applications, 2007, pp. 807-14.

Park, Y., Park, H., Choi, H. y Baik, J., "A Study on the Application of Six Sigma Tools to PSP/TSP for Process Improvement", Proceedings - $5^{\text {th }}$ IEEE/ACIS Int. Conf. on Comput. and Info. Sci., ICIS 2006. In conjunction with $1^{\text {st }}$ IEEE/ACIS, Int. Workshop Component-Based Software Eng., Softw. Archi. and Reuse, COMSAR, 2006.

Pérez, C., Qué significa CMMI. Elementos para la administración cuantitativa, 2012. Recuperado de http://goo.gl/8dq9D

Radice, R., "Statistical Process Control in Level 4 and 5 Organizations Worldwide", Proceedings of the $12^{\text {th }}$ Annual Software Technology Conference, 2000.
Tarhan, A. y Demirörs, O., Investigating Suitability of Software Process and Metrics for Statistical Process Control", Lecture Notes in Computer Science, Vol. 4257, 2006, pp. 88-99.

Vijaya, G. y Arumugam, S., "Monitoring the Stability of the Processes in Defined Level Software Companies Using Control Charts with Three Sigma Limits", WSEAS Transactions on Information Science and Applications, Vol. 7, No. 10, 2010, pp. 1230-39.

VSN International, Genstat, 2012. Recuperado de http://goo.gl/KB66N

Xiaosong, Z., Zhen, H., Fangfang, G. y Shenqing, Z., "Research on the Application of Six Sigma in Software Process Improvement", Proceedings - $20084^{\text {th }}$ International Conference on Intelligent Information Hiding and Multimedia Signal Processing, IIH-MSP, 2008. 